\title{
Atividades antioxidante e antifúngica dos óleos essenciais de Cochlospermum regium frente à Sclerotinia sclerotiorum e Colletotrichum gloeosporioides
}

\author{
Antonio Carlos Pereira de Menezes Filho, Wendel Cruvinel de Sousa, Carlos Frederico de Souza Castro
}

Instituto Federal Goiano - IFGoiano, Mestrado em Agroquímica, Campus Rio Verde, GO. E-mail: astronomoamadorgoias@gmail.com

\section{Resumo}

O objetivo do trabalho foi avaliar os óleos essenciais de Cochlospermum regium e suas atividades antioxidante e antifúngica frente à Sclerotinia sclerotiorum e Colletotrichum gloeosporioides. Foram coletadas raízes e folhas em indivíduos de $C$. regium em uma área de Cerrado, o material foi processado, pesado e a extração dos óleos se deram pelo método de hidrodestilação em Clevenger. Os óleos tiveram seu rendimento avaliado, bem como as atividades antioxidante e antifúngica frente à $S$. sclerotiorum e $C$. gloeosporioides. Os rendimentos foram de 0,168 e 0,301\% para folhas e raízes; a atividade antioxidante apresentou $100 \%$ de redução do radical DPPH foi de $100 \%$ nas concentrações entre 50 a $30 \mu \mathrm{L} \mathrm{mL}^{-1}$ e a inibição micelial de 14,2 e $60,1 \%$ na concentração de $100 \mu \mathrm{L} \mathrm{mL}^{-1}$ para $S$. sclerotiorum e de 84,4 e $81,3 \%$ para $C$. gloeosporioides para os óleos das raízes e folhas, respectivamente.

Palavras-chave: Cochlospermum regium; atividade antifúngica; óleo essencial; atividade antioxidante.

Antioxidant and antifungal activities of Cochlospermum regium essential oils against Sclerotinia sclerotiorum and Colletotrichum gloeosporioides

Abstract

The objective of this work was to evaluate the essential oils of Cochlospermum regium and its antioxidant and antifungal activities against Sclerotinia sclerotiorum and Colletotrichum gloeosporioides. Roots and leaves were collected in individuals of $\boldsymbol{C}$. regium in a Cerrado area, the material was processed, weighed and extraction of the oils were by the hydroventilation method in Clevenger. The oils had their evaluated yield, as well as the antioxidant and antifungal activities against $S$. sclerotiorum and $C$. gloeosporioides. Yields were 0.168 and $0.301 \%$ for leaves and roots; the antioxidant activity showed $100 \%$ reduction of the DPPH radical was $100 \%$ in the concentrations between 50 and $30 \mu \mathrm{L} \mathrm{mL}^{-1}$ and the mycelial inhibition of 14.2 and $60.1 \%$ in the concentration of $100 \mu \mathrm{L} \mathrm{mL}^{-1}$ for S. sclerotiorum and 84.4 and $81.3 \%$ for C. gloeosporioides for root and leaf oils, respectively.

Keywords: Cochlospermum regium; antifungal activity; essential oil; antioxidant activity.

\section{Introdução}

O domínio Cerrado é o segundo maior em área territorial, apresentando rica fauna e flora endêmica no Brasil. O Cerrado está atrás apenas do bioma Amazônico em área de cobertura florestal e da riqueza de biodiversidade. É neste domínio em especial, nas fitofisionomias cerradão, Cerrado ralo e Cerrado stricto sensu, bem como próximas a áreas antropomorfizadas que o espécimen vegetal Cochlospermum regium conhecido popularmente por (algodão-docerrado) habita. O C. regium pertence à família Bixaceae, apresentando médio porte arbustivo, com longas raízes estando estas bem profundas no solo, como meio de propagação apresenta também floração de coloração amarela com leve aroma, e após a fecundação das flores, surgem frutos com sementes revestidas com material lanoso. Esta espécie vegetal da flora brasileira é considerada fitoterapêutica sendo amplamente 
utilizada pela população rural para o tratamento de inúmeras enfermidades (LEME et al., 2017).

O C. regium como planta fitoterápica, apresenta importantes compostos químicos, pertencentes às classes dos fenóis e óleos essenciais, que são encontrados nas raízes, talos, folhas e flores. Os óleos essenciais apresentam em sua composição química altos teores quantitativos de terpenóides, em especial para classe de sesquiterpenos como o $\beta$-copaen-4- $\alpha$-ol e Viridiflorol (INÁCIO et al., 2014).

Os óleos essenciais são compostos químicos voláteis naturais, apresentando complexos compostos como monoterpenos, diterpenos e sesquiterpenos, onde na maioria apresentam odores, e na temperatura ambiente são líquidos. Os óleos essenciais são compostos formados a partir dos metabólitos secundários das plantas, desempenhando funções específicas, como na atração de polinizadores, na proteção da planta contra altas temperaturas, e na defesa contra o ataque de insetos, animais herbívoros e fitopatógenos (PRINS et al., 2006).

Vários compostos químicos que compõem os óleos essenciais apresentam ações antioxidantes, antibacteriana, antifúngica, inseticida, larvicida, leishmanicida dentre outros, sendo de grande importância para as indústrias alimentícia, farmacêutica e de produtos agrícolas, nesta última principalmente no combate a fungos causadores de perdas de produção em grãos e frutas durante a produção e armazenamento todos os anos, gerando significativa redução na economia em milhões de dólares (DIAS et al., 2019; SCHERER et al., 2009).

O mofo-branco (Sclerotinia sclerotiorum) e a antracnose (Colletotrichum gloeosporioides) são doenças fitopatológicas que afetam a produção principalmente nas culturas de soja, milho, feijão e grãos-de-bico, bem como de frutas ainda na lavoura como na pós-colheita do abacate, banana, morango e mamão. Podem ocorrer também em outros órgãos vegetais como folhas, flores e galhos sendo estes dois fitopatógenos frequentes no Brasil (SILVA et al. 2018). O controle de ambas as espécies fúngicas necessita do uso maciço de agentes fungistáticos sintéticos sendo aplicadas várias vezes nas lavouras, com isso, o uso descontrolado de agentes fungicidas ocasiona o desenvolvimento de resistência aos princípios ativos dos antifúngicos comerciais, além disso, ocasionam descontrole ambiental na fauna, contaminação de solos, leitos de rios e sérios problemas para a saúde humana (CRUZ et al. 2010).

Com isso, alternativas naturais como o uso de óleos essenciais a base de metabólitos secundários produzidos pelas plantas, vem se tornando opção alternativa devido a inúmeros compostos químicos que apresentam atividade antifúngica natural, como a piperitona, terpinen4-ol, $\beta$-cariofileno, $\alpha$-humuleno, germacreno D e miristicina (VALADARES et al., 2018).

Verificando a necessidade de novos estudos com base na biodiversidade florística do Cerrado brasileiro, este estudo objetivou avaliar as atividades antioxidante e antifúngica dos óleos essenciais das raízes e folhas de Cochlospermum regium frente às linhagens fúngicas de Sclerotinia sclerotiorum e de Colletotrichum gloeosporioides.

\section{Material e Métodos}

Foram coletadas raízes e folhas em indivíduos de $C$. regium, nas primeiras horas do dia compreendido entre as 6-9 horas da manhã em uma área de Cerrado ralo no município de Rio Verde/GO, Brasil, localizado nas seguintes coordenadas geográficas: $17^{\circ} 47^{\prime} 13.7^{\prime \prime} \mathrm{S}$ $50^{\circ} 57^{\prime} 60.0^{\prime \prime} \mathrm{W}$. Uma exsicata foi herborizada e depositada no Herbário do Instituto Federal Goiano, com a seguinte identificação HRV: 844 .

Para extração dos óleos essenciais foram utilizadas alíquotas de $100 \mathrm{~g}$ de material in natura, sendo em seguida, triturados em processador doméstico com $500 \mathrm{~mL}$ de água destilada e deionizada. Para a obtenção dos óleos essenciais, foi utilizado aparelho de Clevenger com refluxo por 4 horas. Em seguida o hidrolato foi coletado e transferido para um funil de separação sendo lavado com 3 partes de diclorometano. A parte diclorometânica foi reunida em um béquer onde foi seco com sulfato de sódio anidro, e logo após, filtrado em papel de filtro quantitativo. O solvente foi evaporado naturalmente em local escuro em temperatura de $25{ }^{\circ} \mathrm{C}$. O rendimento de óleo essencial foi determinado pela equação 1 .

(\%) $\mathrm{OE}=$ Moe $(\mathrm{g}) / \mathrm{Mam}(\mathrm{g}) \times 100$

Eq. 1

Onde: Moe = massa de óleo essencial extraído, Mam = massa amostra úmida e 100 fator de conversão para porcentagem.

$A$ atividade antioxidante foi determinada conforme Mezza et al. (2018) adaptado. Utilizouse 0 radical livre DPPH (2,2-difenil-1picrilhidrazil). $O$ ensaio antioxidante foi realizado pelo método de microdiluição em microplacas de 
96 poços. Para cada poço foram adicionados 100 $\mu \mathrm{L}$ de uma solução diclorometânica de DPPH na concentração $0,06 \mathrm{mM}, 100 \mu \mathrm{L}$ de óleo essencial diluído em diclorometano nas seguintes diluições com concentrações de 50; 40; 30; 20; 10; 5; 2,5 e $1,25 \mu \mathrm{L} \mathrm{mL}$. As leituras foram realizadas no comprimento de ondas em $517 \mathrm{~nm}$, após $1 \mathrm{~h}$. de reação. Uma curva padrão foi realizada com a solução de DPPH na concentração 0,06 mM. Como controle negativo utilizou-se a solução mãe de DPPH 0,06 mM, e como branco o diclorometano. Os resultados foram expressos em percentagem de redução de DPPH (\%).

Para verificação da atividade antifúngica, foram utilizados isolados de Sclerotinia sclerotiorum obtido de escleródios formados no interior da haste de soja, provenientes de campos comerciais no município de Rio Verde-GO, já para Colletotrichum gloeosporioides o isolado foi obtido diretamente dos frutos de mamoeiros com sintomas de contaminação. As culturas em meio BDA foram doadas pelo laboratório de Produtos Naturais do Instituto Federal Goiano, Campus Rio Verde.

A avaliação dos óleos essenciais de $C$. regium sobre o crescimento micelial de $S$. sclerotiorum e C. gloeosporioides, partiram de concentrações de 100 (óleo puro); 50; 25; 12,5; 6,25; 3,13 e 1,56 $\mathrm{L} \mathrm{mL}^{-1}$ de óleo essencial diluído em DMSO. Como controle negativo, utilizou-se a testemunha (ausência de óleo essencial) e DMSO, e como controle positivo o fungicida Frowncide na concentração de $10 \mu \mathrm{L} \mathrm{mL}^{-1}$. As concentrações dos óleos essenciais foram adicionadas ao meio de cultura BDA em todas as concentrações, bem como para os tratamentos com fungicida comercial e DMSO. Após solidificação do meio, em câmara de fluxo laminar, 1 disco de micélio de S. sclerotiorum e ou C. gloeosporioides com 7 $\mathrm{mm}$ de diâmetro, foi depositado no centro da placa de Petri com $9 \mathrm{~cm}$ de diâmetro. Em seguida foram incubadas à temperatura entre $20^{\circ} \mathrm{C}$, durante 48 horas como descrito por Garcia et al. (2012) adaptado para S. sclerotiorum e de $25^{\circ} \mathrm{C}$ por 10 dias para C. gloeosporioides conforme descrito por Silva et al. (2018) adaptado.

A avaliação consistiu em medições diárias do diâmetro das colônias, por meio de um paquímetro manual, iniciadas após 24 horas do início da incubação e encerradas com tempo de 48 e 240 horas após, quando as colônias fúngicas, do tratamento testemunha, atingiram completamente a área interna da placa. A determinação da porcentagem de inibição de crescimento micelial foi realizada conforme equação 2, proposto por Garcia et al. (2012).

$\mathrm{PIC}=(\mathrm{DTT}-\mathrm{DTQ}) / \mathrm{DTT} \times 100$ Eq. (2)

Onde: $\mathrm{PIC}=$ porcentagem de inibição do crescimento micelial, DTT = diâmetro no tratamento testemunha, DTQ = diâmetro no tratamento químico.

A análise estatística consistiu em triplicatas para o rendimento de óleo essencial e quadruplicata para o ensaio antifúngico, seguido de $( \pm)$ desvio padrão. Os dados foram avaliados pelos testes de Student e Tukey com nível de significância $(p \leq 0,05)$.

\section{Resultados e Discussão}

$\mathrm{Na}$ Tabela 1 está apresentado o rendimento de óleo essencial extraído das raízes e folhas de $C$. regium. Os óleos essenciais apresentaram coloração incolor em ambas as amostras. O rendimento apresentou em ambos os óleos apresentaram diferença significativa em especial para o óleo essencial das raízes com 0,301\%, seguido do óleo essencial extraído das folhas de $0,168 \%$. A espécie C. regium apresentam boa taxa de rendimento com base em $100 \mathrm{~g}$ de massa in natura.

Tabela 1. Rendimento dos óleos essenciais de C. regium, extraídos das raízes e folhas.

\begin{tabular}{lc}
\hline Amostras & Rendimento (\%) \\
\hline OEF & $0,168 \pm 0,02^{\mathrm{b}}$ \\
OER & $0,301 \pm 0,02^{\mathrm{a}}$ \\
\hline
\end{tabular}

OEF: Óleo Essencial Folhas e OER: Óleo Essencial Raiz. Médias seguidas de ( \pm ) desvio padrão. Os resultados foram submetidos ao teste de Student $(p \leq 0,05)$. 
Silva et al. (2019) encontraram rendimento de $0,6 \%$ de óleo essencial em $M$. paniculata. Valadares et al. (2018) obtiveram rendimento de 0,42 e 0,34\% de óleo essencial das inflorescências e folhas de $P$. aduncum. Já Prins et al. (2006), avaliaram o óleo essencial das folhas de $R$. officinalis em duas velocidades onde encontraram rendimentos de 1,0 a 1,07\%. Na Tabela 2 estão apresentados os resultados da atividade antioxidante $\mathrm{EC}_{50}$ dos óleos essenciais das raízes e folhas de $C$. regium.

Tabela 2. Atividade antioxidante $\mathrm{EC}_{50}$, avaliado nos óleos essenciais das raízes e folhas de $C$. regium.

\begin{tabular}{lcc}
\hline Conc. $\boldsymbol{\mu \mathrm { mL } ^ { - 1 }}$ & \multicolumn{2}{c}{ Atividade Antioxidante (\%) } \\
\hline & OER & OEF \\
\cline { 2 - 3 } $\mathbf{0}$ & $100 \pm 0,00^{\mathrm{a}}$ & $100 \pm 0,00^{\mathrm{a}}$ \\
$\mathbf{3 0}$ & $100 \pm 0,00^{\mathrm{a}}$ & $100 \pm 0,00^{\mathrm{a}}$ \\
$\mathbf{2 0}$ & $96,12 \pm 0,03^{\mathrm{a}}$ & $100 \pm 0,00^{\mathrm{a}}$ \\
$\mathbf{1 0}$ & $70,17 \pm 0,01^{\mathrm{c}}$ & $88,75 \pm 0,02^{\mathrm{b}}$ \\
$\mathbf{5}$ & $55,4 \pm 0,01^{\mathrm{d}}$ & $49,55 \pm 0,02^{\mathrm{c}}$ \\
$\mathbf{2 , 5}$ & $22,11 \pm 0,04^{\mathrm{e}}$ & $33,13 \pm 0,04^{\mathrm{c}}$ \\
$\mathbf{1 , 2 5}$ & $9,45 \pm 0,00^{\mathrm{f}}$ & $24,12 \pm 0,01^{\mathrm{d}}$ \\
$\mathbf{0 , 6 2 5}$ & - & $20,10 \pm 0,00^{\mathrm{d}}$ \\
\hline
\end{tabular}

OER: Óleo Essencial Raiz e OEF: Óleo Essencial Folhas. Médias seguidas de ( \pm ) desvio padrão. Letras diferentes na mesma coluna apresentam diferença significativa pelo teste de Tukey à $(p \leq 0,05)$.

Em relação ao ensaio do sequestro do radical livre DPPH, observou-se redução entre as concentrações de 50 a $30 \mu \mathrm{L} \mathrm{mL}^{-1}$ de $100 \%$. A menor atividade observada no óleo essencial das raízes foi de $13,06 \%$ na concentração de $0,625 \mu \mathrm{L}$ $\mathrm{mL}^{-1}$ em uma quantidade de amostra necessária para reduzir em $50 \%$ a concentração inicial de DPPH. Os óleos essenciais de $C$. regium apresentam importante potencial antioxidante. Possivelmente nos óleos essenciais de $C$. regium apresentam compostos químicos que estão associados aos compostos fenólicos tendo 0 potencial antioxidante aumentado como observado na Tabela 2. Xavier et al. (2016) avaliaram o óleo essencial das folhas de $C$. calophyllum onde obtiveram resultado de $34,4 \%$ de redução do radical DPPH, sendo um baixo potencial antioxidante. Silvestri et al. (2010) encontraram redução do radical DPPH de $95,6 \%$ para o óleo essencial de E. caryophyllata.

$\mathrm{Na}$ Figura 1 observa-se os porcentuais de inibição em diferentes concentrações $\left(\mu \mathrm{L} \mathrm{mL}{ }^{-1}\right)$ dos óleos essenciais das raízes e folhas de $C$. regium frente à $S$. sclerotiorum. 
Figura 1. Percentual de inibição de crescimento micelial (PIC \%) de S. sclerotiorum em diferentes concentrações dos óleos essenciais da raiz e folhas de $C$. regium.

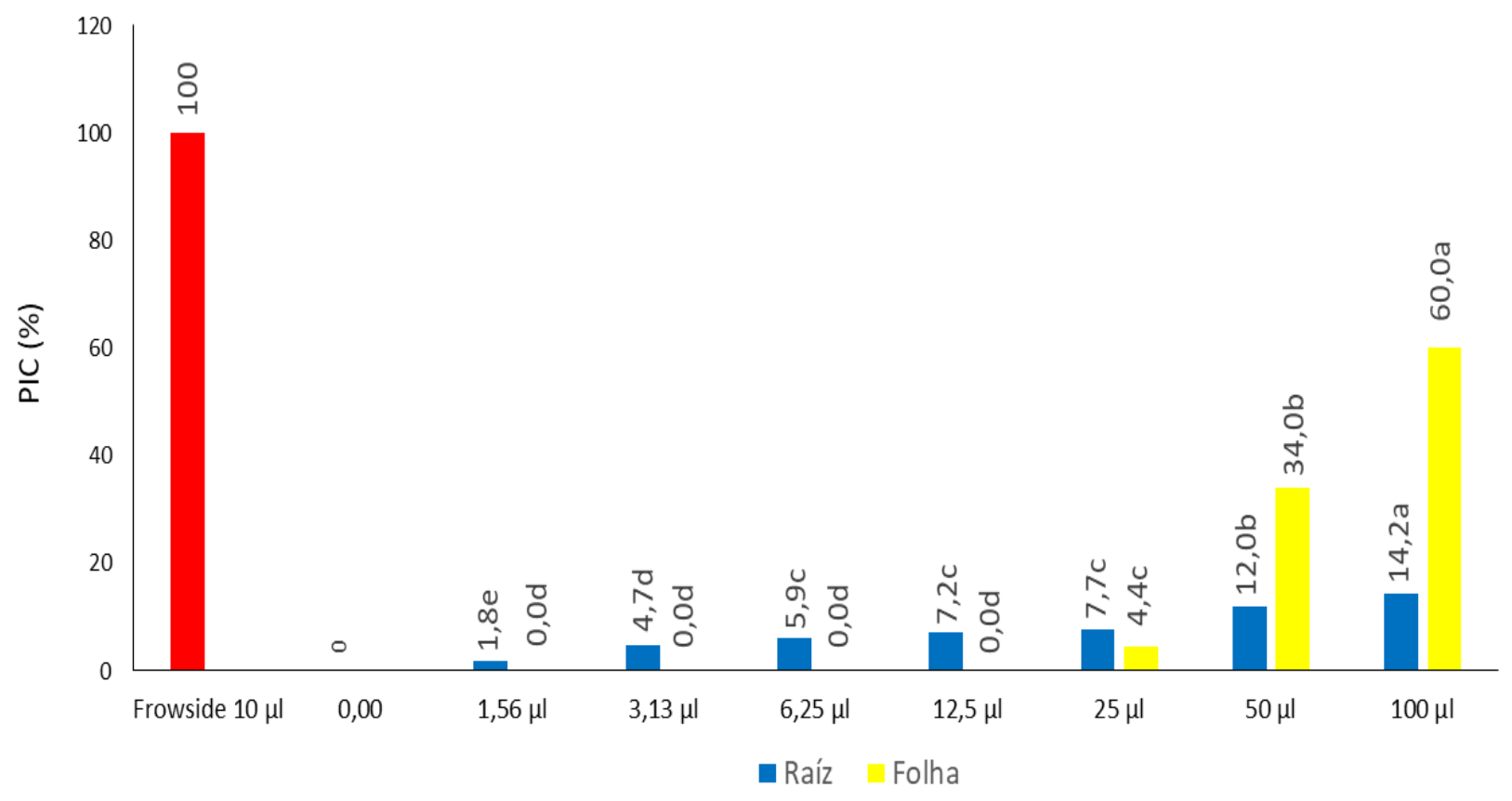

As maiores taxas de inibição micelial de $S$. sclerotiorum foram observados na maior concentração de $100 \mu \mathrm{L} \mathrm{mL}^{-1}$ com taxas de 60,1 e 14,2\% para o óleo essencial das folhas e raízes, respectivamente, e de $100 \%$ quando comparado ao fungicida comercial Frowside na concentração de $10 \mu \mathrm{L} \mathrm{mL}^{-1}$. Os óleos essenciais das folhas e raízes apresentaram estatisticamente três grupos distintos. A menor concentração de inibição para o óleo essencial das folhas foi observado na concentração de $25 \mu \mathrm{L} \mathrm{mL}$, não apresentando inibição nas demais concentrações, e para as raízes, de $1,56 \mu \mathrm{L} \mathrm{mL}^{-1}$ com $1,8 \%$. O óleo essencial das folhas apresentou-se eficiente nas concentrações de 100 e $50 \mu \mathrm{L} \mathrm{mL}^{-1}$. Já o óleo essencial das raízes não foi eficiente como agente fungistático, apresentando máximo de 14,2\% de inibição micelial contra S. sclerotiorum.

Estudo realizado por Silva et al. (2019) utilizando o óleo essencial das folhas de $M$. paniculata, obtiveram na maior concentração de $300 \mu \mathrm{L} \mathrm{mL}^{-1}$ inibição micelial em S. sclerotiorum de $91,2 \%$ e na menor concentração de $12,5 \mu \mathrm{L}$ $\mathrm{mL}^{-1}$ de 28,0\%. Já Xavier et al. (2016) encontraram para o óleo essencial das folhas de C. calophyllum boa eficiência de inibição micelial em S. sclerotiorum nas concentrações de 300 , 200 e $150 \mu \mathrm{L} \mathrm{mL}^{-1}$ iguais a 87,$63 ; 81,33$ e $72,84 \%$ respectivamente.

Na Figura 2 observa-se os percentuais de inibição micelial em $\mathrm{S}$. sclerotiorum em diferentes concentrações $\left(\mu \mathrm{L} \mathrm{mL}^{-1}\right)$ dos óleos essenciais das raízes e folhas de $C$. regium. 
Figura 2. Percentual de inibição de crescimento micelial (PIC \%) de C. gloeosporioides em diferentes concentrações dos óleos essenciais da raiz e folhas de $C$. regium.

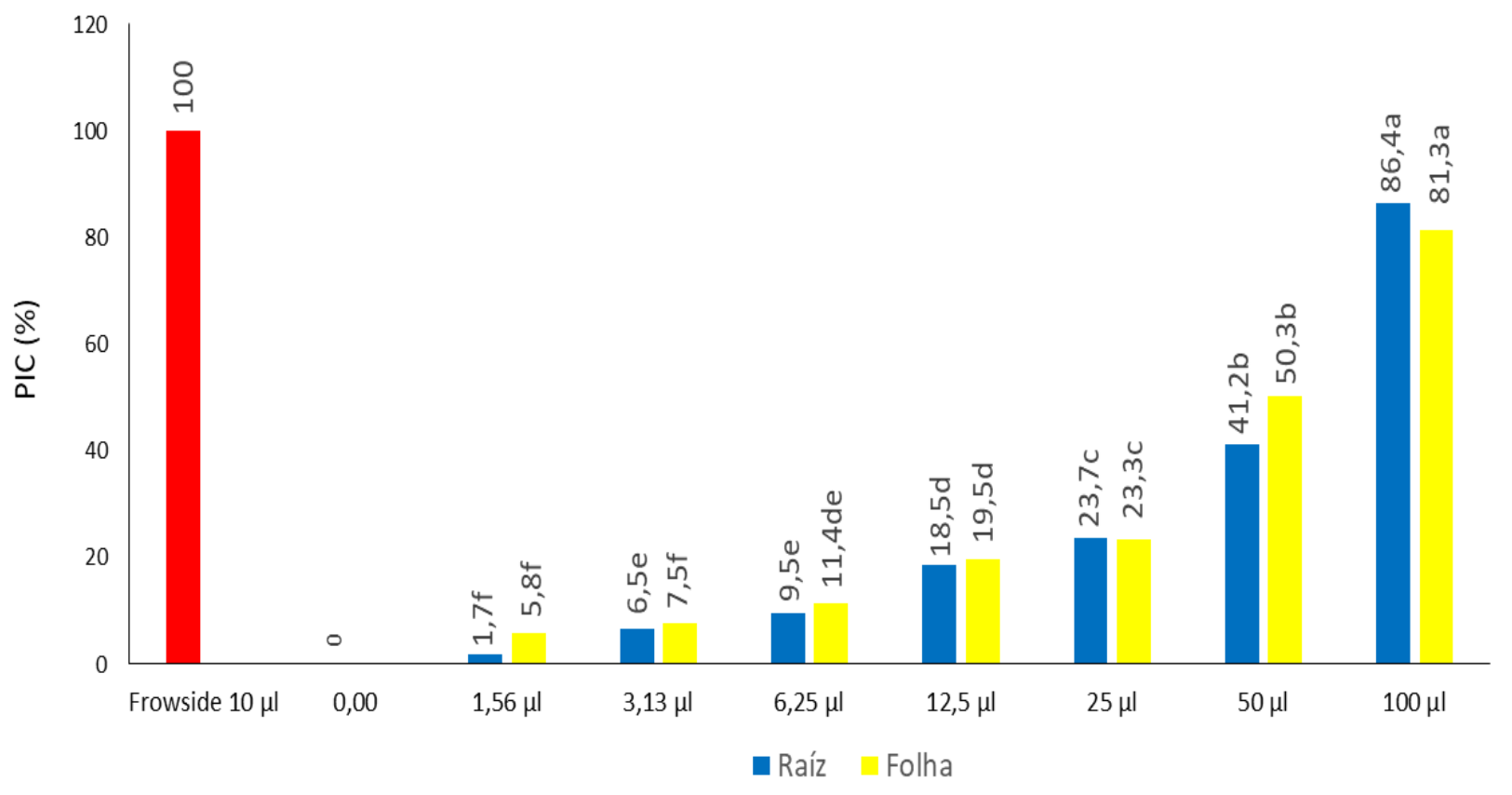

Em relação à percentagem de inibição do crescimento micelial de C. gloeosporioides, comparando as concentrações avaliadas para ambos os óleos essenciais das raízes e folhas de C. regium, é possível verificar diferenças significativas para as concentrações de 100 e 50 $\mu \mathrm{L} \mathrm{mL}{ }^{-1}$ para o óleo das raízes com 86,4 e $41,2 \%$, e para o óleo das folhas nas concentrações de $100,50,25,12,5 \mu \mathrm{L} \mathrm{m}^{-1}$ com 81,3; 50,3; 23,3 e $19,5 \%$ respectivamente. Em especial, a concentração de $100 \mu \mathrm{L} \mathrm{mL}^{-1}$ apresentou maior eficiência quando comparado ao fungicida Frowside com $100 \%$ de inibição do crescimento micelial de $C$. gloeosporioides.

Dias-Arieira et al. (2010) encontraram significativas atividades de inibição em $C$. acutatum avaliando os óleos de nim e de eucalipto com porcentagem de inibição de 84,4 e de $91,1 \%$, respectivamente. Já Souza Júnior et al. (2009) encontraram para os óleos de alecrimpimenta, alfavaca-cravo, capim-santo e cidrão com atividade de $100 \%$ e de goiaba variando entre 44 a $69 \%$ contra o desenvolvimento de $C$. gloeosporioides comprovando o efeito fungicida dos óleos essenciais e não somente a ação fungistática. Os pesquisadores ainda discutem sobre os compostos químicos presentes nos óleos essenciais como promotores da ação de inibição do desenvolvimento micelial, onde, por exemplo, o óleo de alfavaca-cravo apresenta teor de $73,3 \%$ de eugenol, $12,1 \%$ de 1,8 cineol, $2,3 \%$ de $\beta$ - cariofileno e 2,1\% de (Z)-ocimento que são compostos com ação fungicida comprovada.

\section{Conclusão}

Este estudo demonstrou que os óleos essenciais das raízes e folhas de Cochlospermum regium apresentaram bons rendimentos de extração dos óleos e satisfatória atividade antioxidante na redução do radical livre DPPH, bem como excepcional antifúngico in vitro contra S. sclerotiorum e C. gloeosporioides. Os resultados obtidos neste estudo para os óleos essenciais, futuramente poderão ser testados aplicando estes compostos químicos voláteis em experimentos em casas de vegetação e em campo, utilizando os dois fungos em ensaios fungicidas, observando como os dois óleos essenciais se comportam em ambiente controlado e natural.

\section{Agradecimentos}

Ao Instituto Federal de Educação, Ciência e Tecnologia Goiano, Campus Rio Verde; aos Laboratórios de Produtos Naturais; de Química Tecnológica e de Biomoléculas e Bioensaios; a CAPES, CNPq, FINEP e FAPEG pela bolsa de mestrado em Agroquímica para o primeiro autor Antonio.

\section{Referências}

CRUZ, M. J. D. S.; CLEMENTE, E.; CRUZ, M. E. D. S.; MORA, F.; COSSARO, L.; PELISSON, N. Effects of 
bioactive natural compounds on the postharvest Conservation of mango fruits cv. Tommy Atkins. Science and Agrotechnology, v. 34, n. 2, p. 428433, 2010. http://dx.doi.org/10.1590/S141370542010000200022

DIAS, A. L. B.; SOUSA, W. C.; BATISTA, H. R. F.; ALVES, C. C. F.; SOUCHIE, E. L.; SILVA, F. G.; PEREIRA, P. S.; SPERANDIO, E. M.; CAZAL, C. M.; FORIM, M. R.; MIRANDA, M. L. D. Chemical composition and in vitro inhibitory effects of essential oils from fruit peel of three Citrus species and limonene on mycelial growth of Sclerotinia sclerotiorum. Brazilian Journal of Biology, p. 1-5, 2019. https://doi.org/10.1519$\underline{6984.216848}$

DIAS-ARIEIRA, C. R.; FERREIRA, L. R.; ARIEIRA, J. O.; MIGUEL, E. G.; DONEGA, M. A.; RIBEIRO, R. C. F. Atividade do óleo de Eucalyptus citriodora e Azadirachta indica no controle de Colletotrichum acutatum em morangueiro. Summa Phytopathology, v. 36, n. 3, p. 228-232, 2010. https://doi.org/10.1590/S0100-

$\underline{54052010000300007}$

GARCIA, R. Á.; JULIATTI, F. C.; BARBOSA, K. A. G.; CASSEMIRO, T. A. Atividade antifúngica de óleo e extratos vegetais sobre Sclerotinia sclerotiorum. Bioscience Journal, v. 28, n. 1, p. 48-57, 2012.

INÁCIO, M. C.; PAZ, T. A.; BERTONI, B. W.; VIEIRA, M. A. R.; MARQUES, M. O.; PEREIRA, A. M. S. Histochemical investigation of Cochlospermum regium (Schrank) Pilg. leaves and chemical composition of its essential oil. Natural Product Research, v. 28, n. 10, p. 727-731, 2014. https://doi.org/10.1080/14786419.2013.879133

MEZZA, G. N.; BORGARELLO, A. V.; GROSSO, N. R.; FERNANDEZ, H.; PRAMPARO, M. C.; GAYOL, M. F. Antioxidant activity of rosemary essential oil fractions obtained by molecular distillation and their effect on oxidative stability of sunflower oil. Food Chemistry, v. 242, n. 1, p. 9-15, 2018. https://doi.org/10.1016/i.foodchem.2017.09.042

SCHERER, R.; WAGNER, R.; DUARTE, M. C. T.; GODOY, H. T. Composição e atividade antioxidante e antimicrobiana dos óleos essenciais de cravo-da-índia, citronela e palmarosa. Revista Brasileira de Plantas Medicinais, v. 11, n. 4, p. 442-449, 2009.
http://dx.doi.org/10.1590/S1516-$\underline{5722009000400013}$

SILVA, F. F. A. da.; ALVES, C. C. F.; OLIVEIRA FILHO, J. G.; VIEIRA, T. M.; CROTTI, A. E. M.; MIRANDA, M. L. D. Chemical constituents os essential oil from Murraya paniculata leaves and its Application to in vitro biological control of the fungus Sclerotinia sclerotiorum. Food Science and Technology, AHEAD, p. 1-5, 2019. http://dx.doi.org/10.1590/fst.20218

SILVA, T. K.; BORGES, B. G.; FREITAS, A. S.; SOARES, M. G. O.; FREITAS, E. J.; ALCANTRA, E.; FIGUEIREDO, J. R. M. In Vitro antifungal activity of própolis in Colletotrichum spp. of avocado. Journal of University Vale do Rio Verde, v. 16, n. 3, p. 1-6, 2018.

https://doi.org/10.5892/ruvrd.v16i3.5607

SILVESTRI, J. D. F.; PAROUL, N.; CZYEWSKI, E.; LERIN, L.; ROTAVA, I.; CANSIAN, R. L.; MOSSI, A.; TONIAZZO, G.; OLIVEIRA, D.; TREICHEL, H. Perfil da composição química e atividades antibacteriana e antioxidante do óleo essencial do cravo-da-índia (Eugenia caryophyllata Thunb.).

Revista Ceres, v. 57, n. 5, p. 589-594, 2010.

https://doi.org/10.1590/S0034-

737X2010000500004

SOUZA JÚNIOR, I. T.; SALES, N. L. P.; MARTINS, R. $R$. Efeito fungitóxicos de óleos essenciais sobre Colletotrichum gloeosporioides, isolado do maracujazeiro amarelo. Revista Biotemas, v. 22, n. 3, p. 77-83, 2009.

https://doi.org/10.5007/2175-

$\underline{7925.2009 v 22 n 3 p 77}$

PRINS, C. L.; LEMOS, C. S. L.; FREITAS, S. P. Efeito do tempo de extração sobre a composição e o rendimento do óleo essencial de alecrim (Rosmarinus officinalis). Revista Brasileira de Plantas Medicinais, v. 8, n. 4, p. 92-95, 2006.

VALADARES, A. C. F.; ALVES, C. C. F.; ALVES, J. M.; DEUS, I. P. B.; OLIVEIRA FILHO, J. G.; SANTOS, T. C. L.; DIAS, H. J.; CROTTI, A. E. M.; MIRANDA, M. L. D. Essential oils from Piper aduncum inflorescences and leaves: chemical composition and antifungal activity against Sclerotinia sclerotiorum. Anais da Academia Brasileira de Ciências, v. 90, n. 3, p. 2691-2699, 2018.

http://dx.doi.org/10.1590/0001-

$\underline{3765201820180033}$ 
XAVIER, M. N.; ALVES, J. M.; CARNEIRO, N. S.; SOUCHIE, E. L.; SILVA, E. A. J.; MARTINS, C. H. G.; AMBROSIO, M. A. L. V.; EGEA, M. B.; ALVES, C. C. F.; MIRANDA, M. L. D. Composição química do óleo essencial de Cardiopetalum calophyllum Schltdl. (Annonaceae) e suas atividades antioxidante, antibacteriana e antifúngica. Revista Virtual de Química, v. 8, n. 5, p. 14331448, 2016. https://doi.org/10.21577/1984$\underline{6835.20160101}$ 